In order to fix exactly the true wave-length, we intend to take more interference pictures with étalons of different thickness. Further, by continued observations, we hope to diminish the possible error of our spectrographic measurements.

Auroral Observatory, Tromso.

L. Harang.

Physical Institute, Oslo.

L. VEGARD.

${ }^{1}$ L. Vegard, NatURe, 117,$356 ; 1926$

2 L. Vegard, Geophys. Publ., 9, No. 11; 1932.

${ }^{3} \mathrm{~L}$. Vegard, $Z$. Phy8., 75, 30 ; 1932 .

- L. Vegard, Geophys. Publ., 10, No. 4; 1933

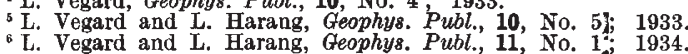

' J. J. Hopfleld, Phys. Rev., 37,$160 ; 1931$.

\section{Isotope Effect in Band Spectra of Hydrides and Deutrides}

The comparison between the band spectra of hydrides and deutrides has shown, as is well known, that the ratio $p^{2}$ of the reduced masses, for example

$$
\rho^{2}=\frac{\mu_{\mathrm{AgH}}}{\mu_{\mathrm{AgD}}}
$$

(where $\mu_{\mathrm{AgH}}=M m_{\mathrm{H}} /\left(M+m_{\mathrm{H}}\right), \quad \mu_{\mathrm{AgD}}=M m_{\mathrm{D}} /(M+$ $\left.m_{\mathrm{D}}\right), m_{\mathrm{H}}=$ mass of the proton, $m_{\mathrm{D}}=$ that of the deuton), calculated in this way, does not agree with the ratio $\rho^{2}$ as deduced from the atomic weights. As a possible explanation, E. Hulthén and W. Holst ${ }^{1}$ have suggested that the electronic system takes part in the rotation and vibration of the molecule and gives a contribution to the effective moment of inertia.

A theoretical discussion may perhaps be interesting. If the hydride (deutride) contains an atom with high atomic number, we can assume the distribution of electrons to be spherically symmetrical and start from the distribution of the corresponding negative ion. A suitable expression for the electronic density is that given by $\mathbf{H}$. Jensen ${ }^{2}$ :

$\mathrm{D}=\frac{N}{4 \pi P_{0} \cdot r_{0}{ }^{3}} \cdot \frac{c^{-X}}{X^{3}}(1+c X)^{3} ; \quad X=\sqrt{\frac{r}{r_{0}}}, r_{0}=\frac{a_{H}}{Z \frac{1}{2} \cdot \lambda}$, $Z=$ atomic number, $N=$ number of electrons, $a_{H}=h^{2} / 4 \pi^{2} m_{e} e^{2}$, where $m_{e}=$ mass of the electron. $P_{0}$ is to be determined by $\int_{0}^{\infty} \mathrm{D} 4 \pi r^{2} d r=N, \lambda$ and $c$ are constants corresponding to $1 / \lambda$ and $c$ in the table of Jensen ${ }^{2}$, and to $\mu$ and $k$ in the table of $\mathrm{Nagy}^{3}$. Calculating the moment of inertia, we get :

$$
I_{e}=m_{e} a_{H^{2}} \times Z^{\frac{1}{3}} \times 7 \cdot 96 \times f\left(\frac{N-Z}{Z}\right)
$$

where $f\left(\frac{N-Z}{Z}\right)$, depending on $\lambda$ and $c$, is a function of $\left(\frac{N-Z}{Z}\right)$ only. If $\left|\frac{N-Z}{Z}\right|<\frac{1}{10}$, which is certain in our case, we can write :

$$
f\left(\frac{N-Z}{Z}\right)=1+3 \cdot 84\left(\frac{N-Z}{Z}\right)+9 \cdot 4\left(\frac{N-Z}{Z}\right)^{2} .
$$

An opportunity for comparison with experimental results is given by the careful investigations ${ }^{4}$ of E. Hulthén and E. Knave on silver hydride and silver deutride. In this case the total angular momentum of the electrons is 0 , and the corrections of Kronig and Van Vleck, being proportional to its square, also equal 0 . So we obtain a lower and an upper limit for the ratio $\rho^{2}$, taken from the rotational structure of the band spectra $\mathrm{AgH}$ and $\mathrm{AgD}, \rho^{2} \mathrm{~min},=$ $\frac{\mu_{\mathrm{AgH}}}{\mu_{\mathrm{AgD}}}=0.50497$, and $\rho^{2} \max .=\frac{\mu_{\mathrm{AgH}}+I_{e} / a^{2}}{\mu_{\mathrm{AgD}}+I_{e} / a^{2}}=$ 0.50545 ( $a=$ nuclear distance). $\rho^{2}$ max. is obtained if all the electrons take part in the rotation.

E. Hulthén and Knave have found $p^{2}=0.50527$ from the $B$-values. Thus the correction is 60 per cent of the theoretical maximum. Using the electronic density $\mathrm{D}$ mentioned above, the calculation shows that this correction will be obtained, provided the four outermost electrons take part in the rotation. This is the same as saying that the electrons outside a sphere with radius $1 \cdot 7 a_{\mathrm{H}}$ are constrained by the hydrogen nucleus to take part in the rotation.

We may verify the reliability of the expression $D$ for the electronic density by calculating the nuclear distance of $\mathrm{AgH}$ to the first approximation. Starting from the charge distribution $D$ of the negative silver ion, we determine the distance from the Ag nucleus at which a positive particle can be in equilibrium. The result, $2 \cdot 78 a_{\mathrm{H}}$, is in good agreement with the experimental value ${ }^{5} \quad 3 \cdot 05 a_{\mathrm{H}}$ and indicates that the moment of inertia will be somewhat greater than our $I_{e}$, if the effect of the hydrogen nucleus on the electronic distribution is taken into account.

I am obliged to Prof. E. Hulthén, who directed my attention to this question, and to Prof. O. Klein, for interesting discussions on the subject.

LAMEK HuLThÉN.

Institutet för mekanik

och matematisk fysik,

Stockholms Högskola. Feb. 12.

${ }^{1}$ Holst and Hulthén, Nature, 133, 496, 796; 1934. Z. Phyя., 90, $712 ; 1934$.

Jensen, Z. Phys., ry, 722; 1932.

Nagy, ibid., 91, 105; 1934

" E. Hulthén and E. Knave, Physica, in press. I am indebted to the authors for being informed of the results before publication.

5 E. Knave, "Dissertation", Stockholm, 1932.

\section{Internal Recombination during Photo-dissociation of Polyatomic Molecules}

IT has been generally admitted that, in the primary decomposition of a molecule by absorption of light, free atoms or unsaturated radicals are produced. In an extensive investigation upon the photodecomposition of carbonyl compounds, Dr. R. G. W. Norrish and his co-workers ${ }^{1}$ have advanced the view that an aldehyde molecule can be disrupted into a saturated hydrocarbon and a carbon monoxide molecule in one process. This is equivalent to a recom. bination of the liberated hydrogen atom and alkyl radical at the moment they leave the remainder of the molecule.

The possibility of such a process can be tested directly if the magnitude of the absorbed quantum is large enough to produce excited atoms, or radicals which will recombine to form an excited molecule emitting its characteristic spectrum. By observing this emission under conditions which prevent the possibility of secondary processes, that is, at very low gas pressures, we should get definite proof of the existence of such an internal recombination.

An investigation in this direction has been undertaken in this laboratory. Polyatomic molecules containing halogen atoms instead of alkyl radicals, 